\section{A FARMÁCIA UNIVERSITÁRIA NO CONTEXTO DAS DIRETRIZES CURRICULARES DO CURSO: UM RELATO DE EXPERIÊNCIA EXITOSA}

Marcelo Silva Silvério José Otávio Do Amaral Corrêa
Introdução: As novas Diretrizes Curriculares Nacionais (DCN) descrevem que a Farmácia Universitária (FU) é cenário obrigatório de prática, visando à execução de atividades de estágio obrigatório. A inserção da FU no processo da formação do farmacêutico tem como grande desafio a integração das necessidades pedagógicas e dos interesses políticos e de gestão das instituições de ensino superior (IES). Objetivo: Relatar a experiência da implantação de uma farmácia regional do município nas dependências da FU da Universidade Federal de Juiz de Fora (UFJF). Resultados: Durante a primeira fase do projeto, até dezembro de 2017, cerca de 18 mil usuários foram atendidos na dispensação e o consultório realizou mais de 800 atendimentos. Nos serviços clínicos, no mesmo período, foram realizados aproximadamente 1200 atendimentos. O projeto, de acordo com dados econômicos do município, em seu $1^{\circ}$ ano de funcionamento, gerou uma redução de aproximadamente $20 \%$ no gasto com medicamentos na região. Os resultados farmacoepidemiológicos e farmacoeconômicos estão sendo investigados. Conclusão: o modelo atual da FU-UFJF reúne os requisitos necessários para as práticas de ensino-aprendizagem no desenvolvimento de competências que abrangem os eixos cuidado em saúde, tecnologia e gestão em saúde previstas nas DCN.

\section{INTRODUÇÃO}

A Farmácia Universitária (FU), de acordo com as premissas definidas pelo Conselho Federal de Farmácia (CFF) e Fórum Nacional de Farmácias Universitárias (FNFU), é um estabelecimento de saúde, que disponibiliza serviços e procedimentos farmacêuticos ao indivíduo, à família e à comunidade, de modo a contribuir para a promoção, proteção e recuperação da saúde, a prevenção de doenças e de outros agravos, e a melhoria dos resultados em saúde ${ }^{1,2}$.

Em 2017, as novas Diretrizes Curriculares Nacionais (DCN) dos cursos de Farmácia descrevem queaFUécenário obrigatório deprática, podendo ser na própria instituição de ensino superior (IES) ou em outro estabelecimento, relacionado à assistência farmacêutica, por meio de convênio, visando à execução de atividades de estágio obrigatório, para todos os estudantes do curso. Ainda de acordo com as DCN os estágios obrigatórios devem contemplar cenários de prática do Sistema Único de Saúde (SUS) nos diversos níveis de complexidade, e deve haver uma integração do curso com o sistema local e regional de saúde do SUS $^{3}$.

Neste contexto, a inserção das FU no processo da formação do farmacêutico tem como grande desafio a integração das necessidades pedagógicas e dos interesses políticos e de gestão das IES.

A Farmácia Universitária da UFJF (FUUFJF) foi fundada em 1995 e até 2014, gerida pela Fundação de apoio da Universidade, desenvolveu atividades magistrais oferecendo a população produtos e serviços de alta qualidade a custos reduzidos. A partir de 2015, a gestão passou a ser integralmente da Universidade por recomendações dos órgãos de controle.

A partir do novo modelo de gestão, foi iniciado com a Prefeitura do município (PJF), um diálogo para a celebração de um convenio que permitisse a integração da FU-UFJF à rede de atenção à saúde. Deste diálogo surgiu o Projeto Farmácia Regional Oeste, um projeto de implantação de uma farmácia regional do município nas dependências da Farmácia Universitária da UFJF. O planejamento da implantação ocorreu entre os anos de 2015-2016 e a inauguração em abril de 2016. A Universidade e a Prefeitura assinaram um acordo de cooperação, onde a FUUFJF disponibiliza a sua estrutura física, recursos humanos e materiais, enquanto a PJF disponibiliza os medicamentos da REMUME e o software de gestão para a dispensação aos usuários do SUS da região.

\section{Aspectos legais e técnicos da FU-UFJF}

Em 2016, no âmbito administrativo, a FU-UFJF foi estabelecida como filial da Universidade através de seu regimento aprovado no Conselho superior, e no âmbito acadêmico foi registrada como um Programa de extensão universitária. A FU-UFJF funciona como farmácia com manipulação o que permite o desenvolvimento de atividades de estágios curriculares e o atendimento a projetos de pesquisa e extensão que demandem produtos magistrais. Atualmente a farmácia não

\section{Universidade Federal de Juiz de Fora}

Recebido: 01/06/2018 Aceito: $25 / 06 / 2018$

Como citar este artigo: Silvério MS e Corrêa JOA. A Farmácia Universitária no contexto das diretrizes curriculares do curso: um relato de experiência exitosa. Rev.

Bras. Farm. Hosp. Serv. Saúde, 9(2): pag-pag, 2019.

Doi: $10.30968 /$ rbfhss.2018.092.008

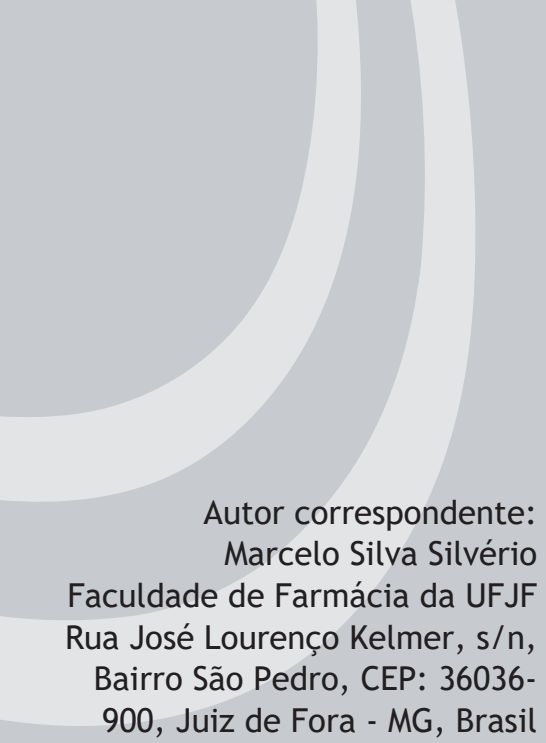


comercializa nenhum tipo de produto diretamente a população. Para a execução de suas atividades e desenvolvimento do convenio a farmácia mantém o seu licenciamento sanitário anual e a sua regularidade junto ao CRF-MG, atendendo as exigências das RDC 67/2007 e RDC 44/2009, entre outras legislações para o setor. O financiamento das atividades da FU-UFJF é realizado com recursos da Faculdade de Farmácia oriundos do orçamento da UFJF.

A equipe de colaboradores é composta por 16 colaboradores, dos quais 10 são servidores ( 5 farmacêuticos, 2 técnicos e 2 auxiliares, e 1 docente coordenador e RT) e 6 são terceirizados. A equipe é responsável pelas atividades técnicas e acadêmicas, incluindo a supervisão dos estágios de graduação e das atividades práticas dos programas de residência farmacêutica da UFJF. Todas as atividades são orientadas por docentes. Em média, ao longo do ano, são atendidos 100 alunos de graduação para estágios, atividades de extensão e pesquisa, e 7 residentes.

\section{O Projeto Farmácia Oeste}

O município possui atualmente 63 unidades básicas de saúde (UBS) distribuídas em 12 regiões sanitárias urbanas e 1 de campo. O projeto se desenvolve na região sanitária oeste que possui 3 UBS. A população estimada, com cobertura de atenção Primária a Saúde (APS) da região é de aproximadamente 50 mil pessoas e corresponde a cerca de $10 \%$ da população coberta no município ${ }^{4}$. Através do projeto, a FU-UFJF dispensa os medicamentos do componente básico da AF e alguns produtos do componente estratégico, aos pacientes atendidos na APS e na Unidade de pronto atendimento (UPA) da região. A FU-UFJF também oferece serviços clínicos de educação em saúde, revisão da farmacoterapia, acompanhamento farmacoterapêutico e conciliação medicamentosa $a^{5,6}$.

A dispensação dos medicamentos é realizada pelos acadêmicos de graduação e residentes em ambiente onde os usuários são atendidos sentados. Durante as orientações para o uso correto dos medicamentos são avaliadas as necessidades dos serviços clínicos individualizados ${ }^{5,7}$. Estes serviços são prestados na sala de serviços farmacêuticos pelos residentes farmacêuticos, de acordo com com o protocolo de atendimento desenvolvido na FU-UFJF 8 .9. Para documentação dos serviços clínicos é utilizado o Sistema REGISTRER (http://www. sistemaregistre.com.br) e a evolução é descrita seguindo a estrutura do modelo SOAP ${ }^{10}$.

\section{A FU-UFJF E A IMPLEMENTAÇÃO DAS NOVAS DCN}

A Farmácia Universitária da UFJF oferece os seguintes serviços clínicos farmacêuticos: dispensação de medicamentos (incluindo orientação sobre o uso correto dos medicamentos, armazenamento e descarte), educação em saúde, revisão da farmacoterapia, conciliação medicamentosa e acompanhamento farmacoterapêutico com procedimentos de aferição de PA, glicemia e temperatura.

O Projeto Farmácia Oeste permitiu o alcance de três fundamentos descritos nas DCN de Farmácia ${ }^{3}$. São eles, a FU como cenário obrigatório de prática, relacionado à assistência farmacêutica, onde se desenvolvem os estágios obrigatórios para todos os estudantes do curso. Segundo, a FU-UFJF é um cenário do SUS e permite a integração do curso com o sistema local e regional de saúde. Este modelo de funcionamento e os serviços desenvolvidos na FU-UFJF, como dispensação, serviços clínicos, manipulação de medicamentos, gestão logística e integração com o SUS, permitem o desenvolvimento de competências que perpassam os 3 eixos de formação descritos nas DCN, o eixo cuidado em saúde, tecnologia e inovação e gestão em saúde.

Neste projeto destaca-se ainda a integração entre estes os diversos atores acadêmicos, professores, farmacêuticos, técnicos, residentes e graduandos, com os usuários do SUS, como demonstrado na figura 1 .

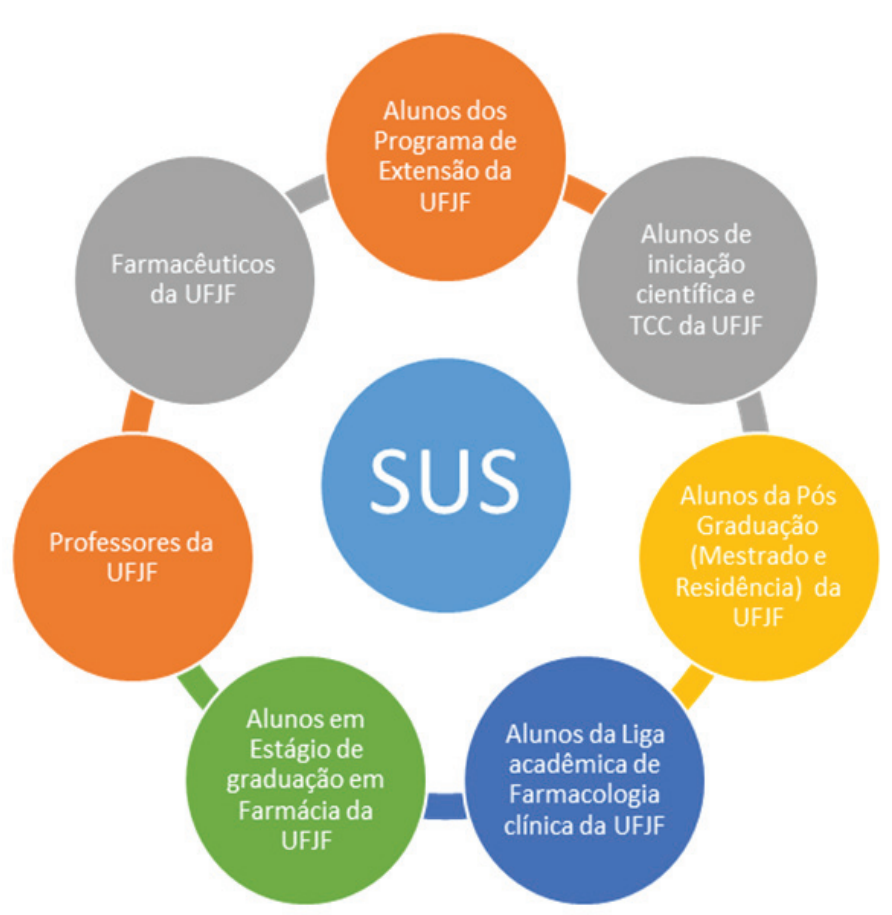

Figura 1: Integração entre os atores envolvidos no Projeto Farmácia Regional Oeste da Farmácia Universitária da UFJF, 2018

\section{RESULTADOS}

O Projeto foi planejado para ser implantado em três fases, sendo a $1^{\text {a }}$ fase a dispensação de medicamentos básicos da REMUME aos usuários de 1 UBS, a 2a fase é a dispensação para usuários de toda a região oeste, e a $3^{\text {a }}$ fase a incorporação de medicamentos estratégicos da REMUME. A primeira fase do projeto, até dezembro de 2017, contou com a participação de 80 alunos, 8 bolsistas, 9 residentes e 6 farmacêuticos. Cerca de 18 mil usuários foram atendidos na dispensação e o consultório realizou mais de 800 atendimentos. Nos serviços clínicos, no mesmo período, foram realizados aproximadamente 1200 atendimentos. O projeto, de acordo com dados econômicos do município, em seu $1^{\circ}$ ano de funcionamento, gerou uma redução de aproximadamente $20 \%$ no gasto com medicamentos na região. Os resultados farmacoepidemiológicos e farmacoeconômicos estão sendo investigados.

$\mathrm{O}$ projeto possui diversos desafios para o seu desenvolvimento e manutenção, entre os quais se destacam o financiamento público da assistência farmacêutica, o financiamento da estrutura da FU-UFJF, a integração efetiva na rede de atenção à saúde no município e o desenvolvimento de um trabalho multiprofissional.

\section{CONCLUSÕES}

O Projeto Farmácia Oeste permitiu a FU-UFJF iniciar uma nova fase de integração com o sistema de saúde pública no município. Neste sentido o modelo atual da FU-UFJF reúne os requisitos necessários para as práticas de ensino-aprendizagem no desenvolvimento de competências que abrangem os eixos cuidado em saúde, tecnologia e gestão em saúde previstas nas DCN. Os desafios são grandes, mas o projeto já demonstrou a capacidade de ampliar o cuidado em saúde e ainda reduzir os gastos no município.

\section{Fontes de Financiamento}

O projeto é financiado pela Universidade Federal de Juiz de Fora através da Faculdade de Farmácia e de programas institucionais de ensino e extensão. Os medicamentos distribuídos são fornecidos pela Prefeitura de Juiz de Fora com financiamento do SUS. 


\section{Conflito de Interesses}

Não há conflito de interesse no desenvolvimento do projeto.

\section{Colaboradores}

MSS: contribuição substancial em todos os aspectos do artigo. JOAC: contribuição em todos os aspectos do artigo.

\section{Agradecimentos}

Agradecimentos aos servidores e alunos da UFJF que atuam na Farmácia Universitária, à Faculdade de Farmácia, ao Departamento de Assistência Farmacêutica da Prefeitura de Juiz de Fora, e a todos os usuários da Farmácia Universitária da UFJF.

\section{REFERÊNCIAS}

1. Conselho Federal de Farmácia (CFF). NOTA TÉCNICA No 01/2016 - a farmácia universitária como indicador obrigatório na avaliação dos cursos de Farmácia. Brasília, 2016: 7p. Disponível em: http://www.cff.org.br/userfiles/file/NT-FU-2016.pdf, acessado em 01/06/2018.

2. Fórum Nacional de Farmácias Universitárias. Farmácia universitária: padrões mínimos/Fórum Nacional de Farmácias, 1ed. Goiânia, UFG, 2017: 48p.

3. Brasil. Ministério da Educação. Conselho Nacional de Educação Câmara de Educação Superior. Resolução nº 6, de 19 de outubro de 2017. Institui as Diretrizes Curriculares Nacionais do Curso de Graduação em Farmácia e dá outras providencias. Diário Oficial da União, Brasília, 20 de outubro de 2017, Seção 1, p. 30. Disponível em: http://portal.mec.gov.br/docman/outubro-2017-pdf/74371-rces006-17-pdf/file, acessado em 01/06/2018.

4. Prefeitura Municipal de Juiz de Fora. Conselho Municipal de Saúde. Resolução 412/2013 - Dispõe sobre o Plano Municipal de Saúde de Juiz de Fora 2014-2017. Juiz de Fora, Conselho Municipal de Saúde, 2013: 123 p. Disponível em: https://www.pjf.mg.gov.br/consehos/cms/arquivos/plano_saude_2014_2017.pdf, acessado em $01 / 06 / 2018$.

5. Júnior JMN. Dispensação: dispensar e entregar não são sinônimos. Uso Racional de Medicamentos: fundamentação em condutas terapêuticas e nos macroprocessos da Assistência Farmacêutica, 2016, 1(16):1-5. Disponível em: https://www.paho.org/bra.../ index.php?option=com_docman\&view=download\&category_ slug=serie-uso-racional-medicamentos-284\&alias=1544-dispensacao-dispensar-e-entregar-nao-sao-sinonimos-4\&Itemid=965, acessado em 01/06/2018.

6. Conselho Federal de Farmácia. Serviços farmacêuticos diretamente destinados ao paciente, à família e à comunidade: contextualização e arcabouço conceitual. Brasília, Conselho Federal de Farmácia, 2016: $200 p$.

7. Conselho Federal de Farmácia. Atividades do Farmacêutico na Farmácia comunitária - Manual III. Brasília, 2009: 15p. Disponível em http://www.cff.org.br/sistemas/geral/revista/pdf/122/encarte_ farmAcia_comunitAria.pdf, acesso em 01/06/2018.

8. BRASIL. Ministério da Saúde. Secretaria de Ciência, Tecnologia e Insumos Estratégicos. Departamento de Assistência Farmacêutica e Insumos Estratégicos. Serviços farmacêuticos na atenção básica à saúde, Caderno 1. Brasília, Ministério da Saúde, 2014: 108p.
9. Hernández DS, Castro MMS, Dáder MJF. Método Dáder - Manual de Seguimento Farmacoterapêutico, 3ed. Lisboa, Edições Universitárias Lusófonas, 2010: 128p. Disponível em: http://pharmcare.pt/ wp-content/uploads/file/Guia_dader.pdf, acesso em 01/06/2018.

10. Cantale CR. História Clinica Orientada a Problemas. Curso Básico de Medicina Familiar, 2003: 14p. Disponível em: https://jaimebueso. files.wordpress.com/2007/07/historia-clinica-orientada-a-problemas.pdf, acesso em 01/06/2018. 